\title{
Heave and pitch motions in shallow water including the effect of forward speed
}

\author{
By ALLEN PLOTKIN \\ Engineering Science Department, California Institute of Technology, Pasadena†
}

(Received 17 June 1976)

The problem of small heave and pitch motions of a slender ship in shallow water including the effect of forward speed is analysed using the method of matched asymptotic expansions. Formulae valid to first order in slenderness are given for the added-mass and damping coefficients in terms of the frequency and subcritical Froude number.

\section{Introduction}

Owing to the increasing size, especially draft, of modern ships and the necessity for them to negotiate water of limited depth, there has been current interest generated in the study of ship motions in restricted waters. According to Beck, Snyder \& Tuck (1974), there are two approaches to the analysis of finite-depth ship motions. First, in the case of moderate depth, a modified strip theory can be used as in Kim (1968). Second, one can assume that the water is shallow and therefore all wavelengths are large compared with the depth. In this approach, which will be pursued here, the depth effect is a dominant one.

Tuck (1970) developed a slender-body theory for ship motions in shallow water at zero forward speed using the method of matched asymptotic expansions, which is applicable when the wavelengths of interest are comparable to the ship length. The solution technique was introduced by Tuck (1966) in his study of a slender ship translating at constant speed in shallow water. Beck \& Tuck $(1971,1972)$ have computed the motions of several ships using the theory.

It is the purpose of this research to extend the results of Tuck (1970) to include the effects of forward speed in the study of heave and pitch motions of a slender ship in shallow water. The solution technique, which uses the method of matched asymptotic expansions, closely parallels Tuck's and added-mass and damping coefficients are obtained as functions of frequency and subcritical Froude number.

\section{Problem formulation}

Consider a rigid slender ship of length $2 l$ translating with speed $U$ and oscillating in pitch and heave. A translating Cartesian co-ordinate system (figure 1) is introduced with its origin in the undisturbed free surface at the mean position of the midship. $x$ is in the direction opposite to the translation and $z$ is positive upwards. With no oscillation,

$\uparrow$ Permanent address: Aerospace Engineering Department, University of Maryland, College Park. 

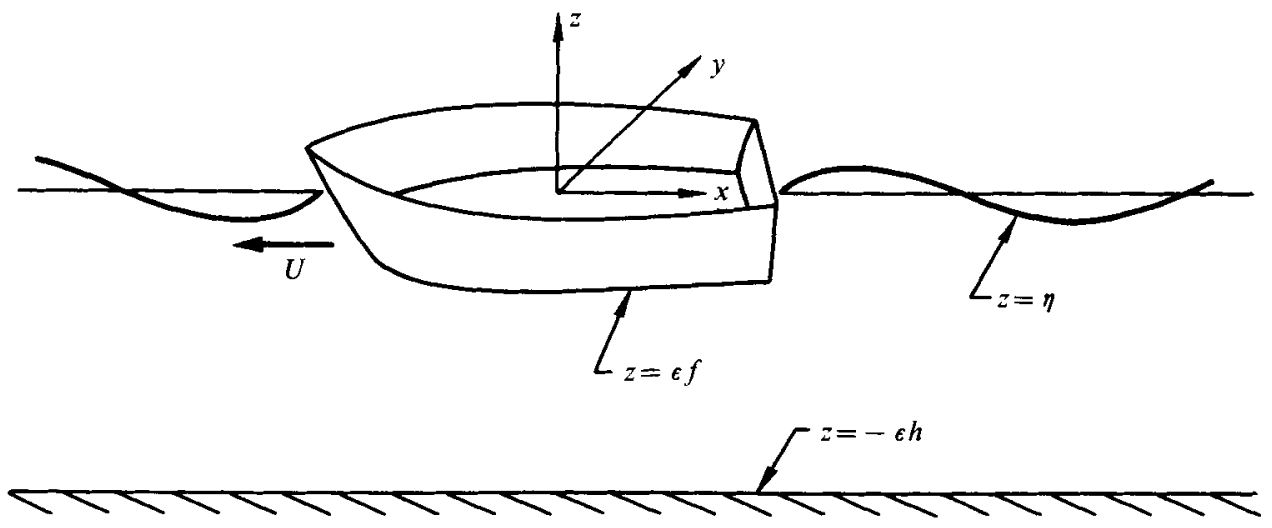

Figure 1. Co-ordinate system.

the ship hull is given by $z=\epsilon f_{0}(x, y)$, where the beam and draft are small, $O(\epsilon)$, with respect to the length. (Note that gauge functions are exhibited explicitly throughout this paper.) Consider harmonic oscillation with frequency $\omega$ and small amplitude, $O(\epsilon \delta)$, where $\delta$ is a small parameter characteristic of the motion. The ship hull is then given by

$$
z=\epsilon f_{0}(x, y)+\xi_{3} \epsilon \delta e^{-i \omega t}-x \xi_{5} \epsilon \delta e^{-i \omega t} \equiv \epsilon f(x, y, t) .
$$

The bottom and free surface are described by

$$
z+\epsilon h=0, \quad z=\eta(x, y, t) .
$$

The water is shallow, so that the undisturbed water depth is $O(\epsilon)$ and the length of all generated waves is much larger than the depth. Following Tuck (1970), we take $\omega l / c$ to be $O(1)$, where $c=(g h \epsilon)^{\frac{1}{2}}$ is the shallow-water phase speed and $g$ is the gravitational acceleration. Following Tuck (1966) we choose the depth Froude number $F=U / c$ to be $O(1)$, which means that the conventional Froude number based on length is small, $O\left(\epsilon^{\frac{1}{2}}\right)$. This is a range where wave making is significant. The flow is subcritical with $F<1$. It is convenient, to keep track of the ordering of terms in the following analysis, to let $U$ and $l$ be $O(1)$ and $g$ be $O\left(\epsilon^{-1}\right)$ (see, for example, Ogilvie 1967).

For incompressible irrotational flow the velocity is represented as the positive gradient of a velocity potential $\phi(x, y, z, t)$ which satisfies Laplace's equation. The complete set of equations is given in Plotkin (1976a) as

$$
\begin{gathered}
\phi_{x x}+\phi_{y y}+\phi_{z z}=0 \text { in fluid domain, } \\
\phi_{z}=0 \text { on } z=-\epsilon h, \\
\epsilon f_{t}+\epsilon U f_{x}+\epsilon \phi_{x} f_{x}+\epsilon \phi_{y} f_{y}-\phi_{z}=0 \text { on } z=\epsilon f, \\
\eta_{t}+U \eta_{x}+\phi_{x} \eta_{x}+\phi_{y} \eta_{y}-\phi_{z}=0 \text { on } z=\eta \\
g \eta+\phi_{t}+U \phi_{x}+\frac{1}{2}\left(\phi_{x}^{2}+\phi_{y}^{2}+\phi_{z}^{2}\right)=0 \text { on } z=\eta .
\end{gathered}
$$

Since $\epsilon$ is an appropriate scale in the vertical direction, the co-ordinate $Z=z / \epsilon$ will be used. The velocity potential and free-surface elevation are split into steady and unsteady parts and only terms linear in $\delta$ are kept:

$$
\begin{gathered}
\phi=\Phi(x, y, Z)+\delta \Psi(x, y, Z) e^{-i \omega t}, \\
\eta=\zeta(x, y)+\delta \theta(x, y) e^{-i \omega t} .
\end{gathered}
$$




\section{Inner expansion}

In the region near the ship, the co-ordinates have the magnitudes $x=O(1)$ and $y, z=O(\epsilon)$. The inner variable $Y=y / \epsilon$ is introduced. The velocity potential is expanded in an asymptotic series in $\epsilon$ :

To $O\left(\epsilon^{2}\right),(4 a)$ becomes

$$
\begin{aligned}
& \Phi=\epsilon \Phi^{(1)}+\epsilon^{2} \Phi^{(2)}+\ldots \\
& \Psi=\epsilon \Psi^{(1)}+\epsilon^{2} \Psi^{(2)}+\ldots
\end{aligned}
$$

$$
\nabla^{2} \Phi^{(1)}=\nabla^{2} \Phi^{(2)}=\nabla^{2} \Psi^{(1)}=\nabla^{2} \Psi^{(2)}=0,
$$

where

while $(4 b)$ becomes

$$
\nabla^{2} \equiv \partial^{2} / \partial Y^{2}+\partial^{2} / \partial Z^{2},
$$

$$
\Phi_{Z}^{(1)}=\Phi_{Z}^{(2)}=\Psi_{Z}^{(1)}=\Psi_{Z}^{(2)}=0 \quad \text { on } \quad Z=-h .
$$

The body boundary condition $(4 c)$ is transferred to the mean hull position $Z=f_{0}$ to yield

$$
\begin{gathered}
\Phi_{N}^{(1)}=\Psi_{N}^{(1)}=0 \\
\Phi_{N}^{(2)}=-U f_{0 x}\left(1+f_{0 Y}^{2}\right)^{-\frac{1}{2}} \\
\Psi_{N}^{(2)}=\left[i \omega\left(\xi_{3}-x \xi_{5}\right)+U \xi_{5}-\left(\xi_{3}-x \xi_{5}\right)\left(f_{0 Y} \Phi_{Y Z}^{(2)}-\Phi_{Z Z}^{(2)}\right)\right]\left(1+f_{0 Y}^{2}\right)^{-\frac{1}{2}}
\end{gathered}
$$

where $\mathrm{N}$ is the outward normal in the $Y, Z$ plane expressed in inner variables. The free-surface conditions $(4 d, e)$ are combined and transferred to $Z=0$ to yield

$$
\Phi_{Z}^{(1)}=\Phi_{Z}^{(2)}=\Psi_{Z}^{(1)}=\Psi_{Z}^{(2)}=0 .
$$

Tuck (1966) has obtained the solution for $\Phi$, which may be written as

$$
\begin{aligned}
& \Phi^{(1)}=\Phi^{(1)}(x), \\
& \Phi^{(2)}=\Phi^{(21)}(x)+\Phi^{(22)}(Y, Z ; x),
\end{aligned}
$$

where $\Phi^{(1)}$ and $\Phi^{(21)}$ are arbitrary and from conservation of mass

$$
\Phi^{(22)} \rightarrow U S^{\prime}(x)|Y| / 2 \hbar+o(1) \text { as }|Y| \rightarrow \infty,
$$

where $\epsilon^{2} S(x)$ is the wetted hull cross-sectional area.

We may write

$$
\begin{aligned}
& \Psi^{(1)}=\Psi^{(1)}(x), \\
& \Psi^{(2)}=\Psi^{(21)}(x)+\Psi^{(22)}(Y, Z ; x),
\end{aligned}
$$

where $\Psi^{(1)}$ and $\Psi^{(21)}$ are arbitrary. The unsteady flux leaving the inner region is obtained by integrating $\Psi_{N}^{(2)}$ in $(9 c)$ around the wetted cross-section. For a ship with symmetry about the centre-plane $Y=0$ and with a vertical tangent at the waterline, use of $(9 b)$ gives the flux as

$$
\text { Flux }=\left\{\left[i \omega\left(\xi_{3}-x \xi_{5}\right)+U \xi_{5}\right] B(x)-U\left(\xi_{3}-x \xi_{5}\right) B^{\prime}(x)\right\},
$$

where $\epsilon B(x)$ is the width of the waterline. A suitable boundary condition as $|Y| \rightarrow \infty$ for $\Psi(22)$ is determined from conservation of mass as

$$
\Psi^{(22)} \rightarrow(\text { Flux })|Y| / 2 h+o(1) .
$$




\section{Outer expansion}

In the region far from the ship, the co-ordinates have the magnitudes $x, y=O(1)$ and $z=O(\epsilon)$. The velocity potential is expanded in an asymptotic series in $\epsilon$ :

$$
\begin{gathered}
\Phi=\epsilon \phi^{(1)}+\epsilon^{2} \phi^{(2)}+\epsilon^{3} \phi^{(3)}+\ldots \\
\Psi=\epsilon \psi^{(1)}+\epsilon^{2} \psi^{(2)}+\epsilon^{3} \psi^{(3)}+\ldots
\end{gathered}
$$

To $O\left(\epsilon^{3}\right),(4 a)$ becomes

while $(4 b)$ becomes

$$
\begin{gathered}
\phi_{Z Z}^{(1)}=\phi_{Z Z}^{(2)}=\psi_{Z Z}^{(1)}=\psi_{Z Z}^{(2)}=0, \\
\phi_{Z Z}^{(3)}=-\phi_{x x}^{(1)}-\phi_{y y}^{(1)}, \quad \psi_{Z Z}^{(3)}=-\psi_{x x}^{(1)}-\psi_{y y}^{(1)},
\end{gathered}
$$

$$
\phi_{Z}^{(1)}=\phi_{Z}^{(2)}=\phi_{Z}^{(3)}=\psi_{Z}^{(1)}=\psi_{Z}^{(2)}=\psi_{Z}^{(3)}=0 \quad \text { on } \quad Z=-h .
$$

If $(17 a-c)$ are integrated once with respect to $Z$ then use of (18) leads to

$$
\begin{gathered}
\phi^{(1)}=\phi^{(1)}(x, y), \quad \phi^{(2)}=\phi^{(2)}(x, y), \quad \psi^{(1)}=\psi^{(1)}(x, y), \quad \psi^{(2)}=\psi^{(2)}(x, y), \\
\phi_{Z}^{(3)}=-(Z+h)\left(\phi_{x x}^{(1)}+\phi_{y y}^{(1)}\right), \quad \psi_{Z}^{(3)}=-(Z+h)\left(\psi_{x x}^{(1)}+\psi_{y y}^{(1)}\right) .
\end{gathered}
$$

The free-surface conditions are combined and transferred to $Z=0$ to yield the following equations:

$$
\begin{gathered}
\beta^{2} \phi_{x x}^{(1)}+\phi_{y y}^{(1)}=0, \\
\beta^{2} \psi_{x x}^{(1)}+\psi_{y y}^{(1)}+\omega^{2} \psi r^{(1)} / c^{2}+2 i \omega F \psi_{x}^{(1)} / c=0,
\end{gathered}
$$

with $\beta^{2}=1-F^{2}$. Equation $(20 a)$ is the equation for the first-order outer potential in Tuck (1966) and (20b) is the reduced wave equation in a translating co-ordinate system and also appears in Plotkin (1976a), which treats the translation of a slender ship over a wavy wall in shallow water.

The equations are solved formally using Green's function source distributions to yield

with

$$
\begin{gathered}
\phi^{(1)}=\int_{-\infty}^{\infty} G(x-\xi, y) \mu(\xi) d \xi \\
\psi^{(1)}=\int_{-\infty}^{\infty} G^{(1)}(x-\xi, y) \mu^{(1)}(\xi) d \xi \\
G(x, y)=(2 \pi \beta)^{-1} \log \left(x^{2}+\beta^{2} y^{2}\right)^{\frac{1}{2}} \\
G^{(1)}(x, y)=-i(4 \beta)^{-1} \exp \left(-i \omega F x / c \beta^{2}\right) H_{0}^{(1)}\left[\omega\left(x^{2}+\beta^{2} y^{2}\right)^{\frac{1}{2}} / c \beta^{2}\right]
\end{gathered}
$$

\section{Matching}

To determine the unknown functions $\Phi^{(1)}, \Psi^{(1)}, \mu$ and $\mu^{(1)}$, the inner and outer expansions are matched using a matching principle from Van Dyke (1975, p. 90). The two-term inner expansion of the one-term outer expansion is

$$
\epsilon \phi^{(1)}(x, 0)+\frac{1}{2} \epsilon \mu(x)|y|+\epsilon \delta \psi^{(1)}(x, 0) e^{-i \omega t}+\frac{1}{2} \epsilon \delta \mu^{(1)}(x)|y| e^{-i \omega t} .
$$

The one-term outer expansion of the two-term inner expansion is

$$
\begin{aligned}
\epsilon \Phi^{(1)}(x) & +U \epsilon S^{\prime}(x)|y| / 2 h+\epsilon \delta \Psi^{(1)}(x) e^{-i \omega t} \\
& +\epsilon \delta(2 h)^{-1}\left[i \omega\left(\xi_{3}-x \xi_{5}\right) B(x)+U \xi_{5} B(x)-U\left(\xi_{3}-x \xi_{5}\right) B^{\prime}(x)\right]|y| e^{-i \omega t}
\end{aligned}
$$


Setting $(22 a)$ equal to $(22 b)$ gives

$$
\begin{gathered}
\Phi^{(1)}(x)=\phi^{(1)}(x, 0), \quad \Psi^{(1)}(x)=\psi^{(1)}(x, 0), \quad \mu(x)=U S^{\prime}(x) / h, \\
\mu^{(1)}(x)=\left[i \omega\left(\xi_{3}-x \xi_{5}\right) B(x)+U \xi_{5} B(x)-U\left(\xi_{3}-x \xi_{5}\right) B^{\prime}(x)\right] h^{-1} .
\end{gathered}
$$

The lowest-order velocity potential in the inner region is therefore

with

$$
\begin{aligned}
& \phi=\epsilon \Phi^{(1)}(x)+\epsilon \delta \Psi^{(1)}(x) e^{-i \omega t}, \\
& \Phi^{(1)}=U(2 \pi \beta h)^{-1} \int_{-\infty}^{\infty} S^{\prime}(\xi) \log |x-\xi| d \xi, \\
& \Psi^{(1)}=-i(4 \beta h)^{-1} \int_{-\infty}^{\infty}\left[i \omega\left(\xi_{3}-\xi \xi_{5}\right) B(\xi)+U \xi_{5} B(\xi)-U\left(\xi_{3}-\xi \xi_{5}\right) B^{\prime}(\xi)\right] \\
& \times \exp \left[-i \omega F(x-\xi) / c \beta^{2}\right] H_{0}^{(1)}\left[\omega|x-\xi| / c \beta^{2}\right] d \xi .
\end{aligned}
$$

$\Phi^{(1)}$ has previously been obtained by Tuck (1966). 'Tuck (1970) has also obtained $\Psi^{(1)}$ for the case $U=0$.

\section{Unsteady hydrodynamic pressure and forces}

To lowest order in $\epsilon$, the unsteady hydrodynamic pressure in the near field is obtained from the linearized Bernoulli equation

$$
p=-\rho\left(\phi_{t}+U \phi_{x}\right)
$$

where $\rho$ is the fluid density and the unsteady part of $\phi$ is

$$
\phi=\epsilon \delta^{(1)}(x) e^{-i \omega t} .
$$

Substitution of (26) into (25) leads to

$$
p=-\rho \epsilon \delta\left(-i \omega \Psi^{(1)}+U \Psi_{x}^{(1)}\right) e^{-i \omega t} .
$$

Note that the unsteady pressure and velocity poteritial depend spatially only on the streamwise co-ordinate $x$. To this order these quantities are constant in cross-sectional planes. The ship geometry appears only through $B(x)$ and its streamwise derivative. There is no effect of underwater geometry. Also, the results depend globally on the axial distribution of $B(x)$ through the Green's function integrals.

The heave force, positive upwards, is

$$
F_{3}=\epsilon \int_{-l}^{l} p(x) B(x) d x
$$

and with an integration by parts and use of (27) becomes

$$
F_{3}=\rho \epsilon^{2} \delta e^{-i \omega t} \int_{-l}^{l}\left[i \omega B(x)+U B^{\prime}(x)\right] \Psi^{(1)}(x) d x
$$

The pitching moment, positive clockwise, is

$$
F_{5}^{\prime}=-\epsilon \int_{-l}^{l} x p(x) B(x) d x=-\rho \epsilon^{2} \delta e^{-i \omega t} \int_{-l}^{l}\left[i \omega x B(x)+U(x B(x))^{\prime}\right] \Psi^{(1)}(x) d x .
$$

Tuck (1970) defines the appropriate components of the transfer matrix $T_{i j}$ by

$$
\begin{aligned}
& F_{3}=\epsilon \delta e^{-i \omega t}\left[\xi_{3} T_{33}+\xi_{5} T_{35}\right] \\
& F_{5}=\epsilon \delta e^{-i \omega t}\left[\xi_{3} T_{53}+\xi_{5} T_{55}\right]
\end{aligned}
$$


The added-mass and damping coefficients $a_{i j}$ and $b_{i j}$, respectively, are related to $T_{i j}$ by

$$
T_{i j}=\omega^{2} a_{i j}+i \omega b_{i j}
$$

With the use of the equations of this section and (24c) and after some manipulation, we obtain $a_{i j}$ and $b_{i j}$ for $i, j=3$ or 5 as

$$
\begin{gathered}
a_{33}=-\rho(4 \beta h)^{-1} \epsilon \int_{-l}^{l} \int_{-l}^{l}\left\{\left[B(x) B(\xi)+U^{2} \omega^{-2} B^{\prime}(x) B^{\prime}(\xi)\right] \cos \right. \\
\left.+U \omega^{-1}\left[B(x) B^{\prime}(\xi)-B(\xi) B^{\prime}(x)\right] \sin \right\} Y_{0} d \xi d x, \quad(33 a) \\
b_{33}=\rho \omega(4 \beta h)^{-1} \epsilon \int_{-l}^{l} \int_{-l}^{l}\left\{\left[B(x) B(\xi)+U^{2} \omega^{-2} B^{\prime}(x) B^{\prime}(\xi)\right] \cos \right. \\
\left.+U \omega^{-1}\left[B(x) B^{\prime}(\xi)-B(\xi) B^{\prime}(x)\right] \sin \right\} J_{0} d \xi d x, \quad(33 b) \\
a_{35}=\rho(4 \beta h)^{-1} \epsilon \int_{-l}^{l} \int_{-l}^{l}\left\{\left[\xi B(\xi) B(x)+U^{2} \omega^{-2} B^{\prime}(x)(\xi B(\xi))^{\prime}\right]\left(Y_{0} \cos -J_{0} \sin \right)\right. \\
\left.\quad+U \omega^{-1}\left[B(x)(\xi B(\xi))^{\prime}-\xi B(\xi) B^{\prime}(x)\right]\left(J_{0} \cos +Y_{0} \sin \right)\right\} d \xi d x, \quad(33 c) \\
b_{35}=-\rho \omega(4 \beta h)^{-1} \epsilon \int_{-l}^{l} \int_{-l}^{l}\left\{\left[\xi B(\xi) B(x)+U^{2} \omega^{-2} B^{\prime}(x)(\xi B(\xi))^{\prime}\right]\left(J_{0} \cos +Y_{0} \sin \right)\right. \\
\left.-U \omega^{-1}\left[B(x)(\xi B(\xi))^{\prime}-\xi B(\xi) B^{\prime}(x)\right]\left(Y_{0} \cos -J_{0} \sin \right)\right\} d \xi d x, \quad(33 d) \\
a_{53}=\rho(4 \beta h)^{-1} \epsilon \int_{-l}^{l} \int_{-l}^{l}\left\{\left[x B(x) B(\xi)+U^{2} \omega^{-2}(x B(x))^{\prime} B^{\prime}(\xi)\right]\left(Y_{0} \cos -J_{0} \sin \right)\right. \\
\left.+U \omega^{-1}\left[x B(x) B^{\prime}(\xi)-B(\xi)(x B(x))^{\prime}\right]\left(J_{0} \cos +Y_{0} \sin \right)\right\} d \xi d x, \quad(33 e) \\
\left.\quad+U \omega^{-1}\left[x B(x) B^{\prime}(\xi)-B(\xi)(x B(x))^{\prime}\right]\left(Y_{0} \cos -J_{0} \sin \right)\right\} d \xi d x, \quad(33 f) \\
b_{53}=-\rho \omega(4 \beta h)^{-1} \epsilon \int_{-l}^{l} \int_{-l}^{l}\left\{\left[x B(x) B(\xi)+U^{2} \omega^{-2} B^{\prime}(\xi)(x B(x))^{\prime}\right]\left(J_{0} \cos +Y_{0} \sin \right)\right. \\
\left.\left.\quad-U(x)(\xi B(\xi))^{\prime}-\xi B(\xi)(x B(x))^{\prime}\right] \sin \right\} J_{0} d \xi d x, \quad(33 h) \\
b_{55}=\rho \omega(4 \beta h)^{-1} \epsilon \int_{-l}^{l} \int_{-l}^{l}\left\{\left[x \xi B(x) B(\xi)+U^{2} \omega^{-2}(x B(x))^{\prime}(\xi B(\xi))^{\prime}\right] \cos \right. \\
a_{55}=-\rho(4 \beta h)^{-1} \epsilon \int_{-l}^{l} \int_{-l}^{l}\left\{\left[x \xi B(x) B(\xi)+U^{2} \omega^{-2}(x B(x))^{\prime}(\xi B(\xi))^{\prime}\right] \cos \right. \\
\left.+U \omega^{-1}\left[x B(x)(\xi B(\xi))^{\prime}-\xi B(\xi)(x B(x))^{\prime}\right] \sin \right\} Y_{0} d \xi d x, \quad(33 g)
\end{gathered}
$$

where

$$
\begin{array}{cl}
\cos =\cos \omega F(x-\xi) / c \beta^{2}, & \sin =\sin \omega F(x-\xi) / c \beta^{2}, \\
J_{0}=J_{0}\left(\omega|x-\xi| / c \beta^{2}\right), & Y_{0}=Y_{0}\left(\omega|x-\xi| c \beta^{2}\right) .
\end{array}
$$

Tuck (1970) has obtained the results $(33 a-h)$ for the case $U=0$.

\section{Discussion}

Consider the added-mass and damping coefficients in $(33 a-h)$. It is noted that the effect of forward speed appears both explicitly and implicitly (through the trigonometric and Bessel functions in the integrands). It is seen that in general the pure heave and pitch coefficients $a_{33}, a_{55}, b_{33}$ and $b_{55}$ are even functions of the forward speed. For a ship with fore-and-aft symmetry, the cross-coupled coefficients are non-zero only 

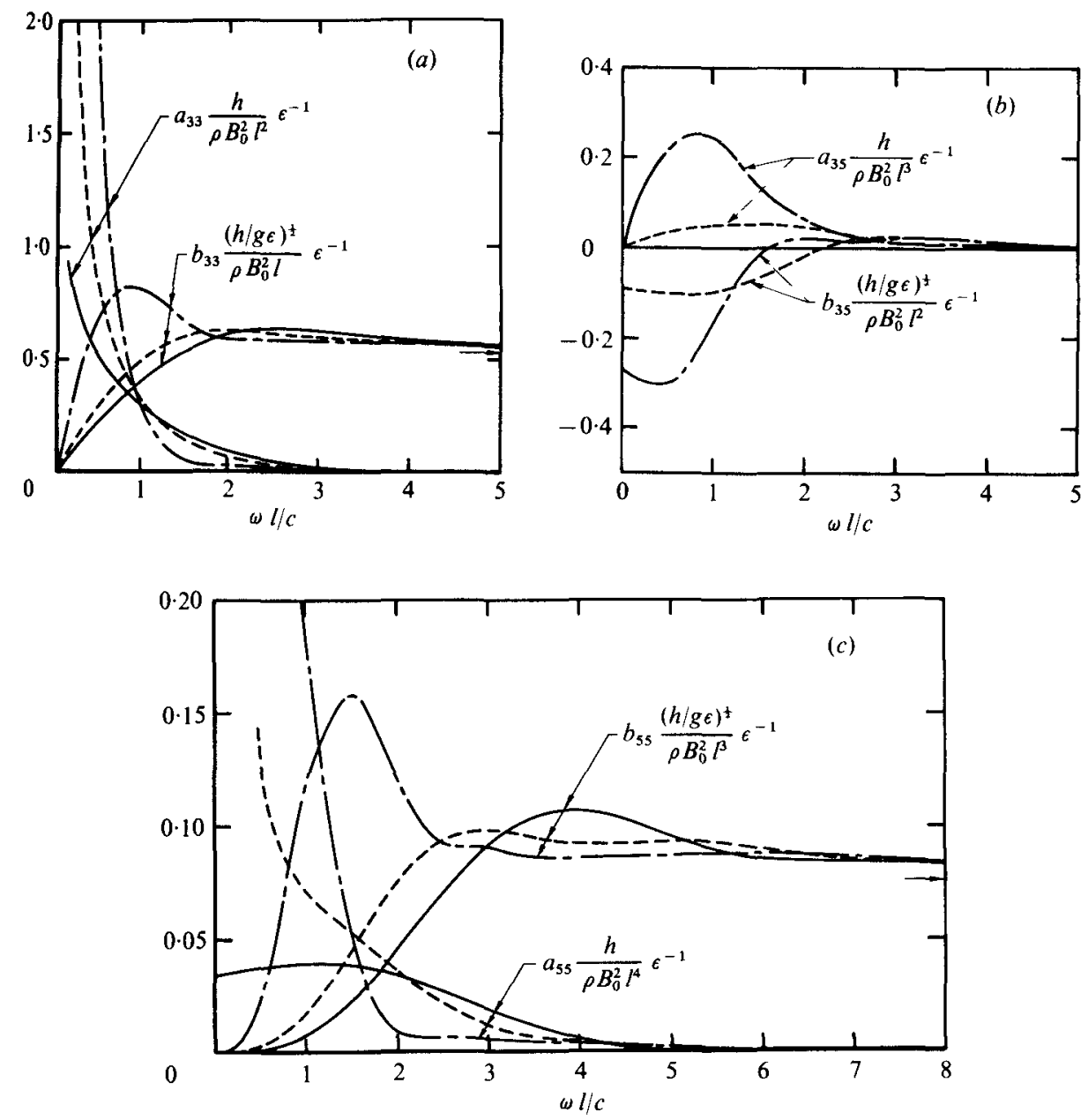

Frgure 2. (a) Heave, (b) heave-pitch and (c) pitch added-mass and damping coefficients for ship with parabolic waterline of beam $\epsilon B_{0}$ and half-length $l$, in water of depth $\epsilon h$, plotted against non-dimensional frequency $\omega l / c .-, F^{\top}=0 ; \cdots, F=0 \cdot 3 ;--, F^{\top}=0 \cdot 6$; $\rightarrow$, high frequenoy limit.

for $U \neq 0$. Also, the cross-coupled coefficients are odd functions of forward speed. For fore-and-aft symmetry, $a_{35}=-a_{53}$ and $b_{35}=-b_{53}$. The above symmetry conditions are identical to those derived for ship oscillations in deep water by Timman \& Newman (1962).

The shallow-water ship-motion theory developed here has the same restrictions on Froude number as the theory of Tuck (1966) and the same restrictions on frequency as the theory of Tuck (1970). The solution is not valid in the neighbourhood of $F=1$, the transcritical region. Lea \& Feldman (1972) have extended the steady theory of Tuck (1966) into this region by solving a nonlinear transonic flow equation instead of $(20 a)$. Pathological behaviour as $F \rightarrow 1$ is expected in the present unsteady theory since the coefficients are proportional to $\left(1-F^{2}\right)^{-\frac{1}{2}}$.

It is noted that the coefficients in $(33 a-h)$ become independent of both forward speed and frequency as the frequency becomes large. The high frequency limit of the 

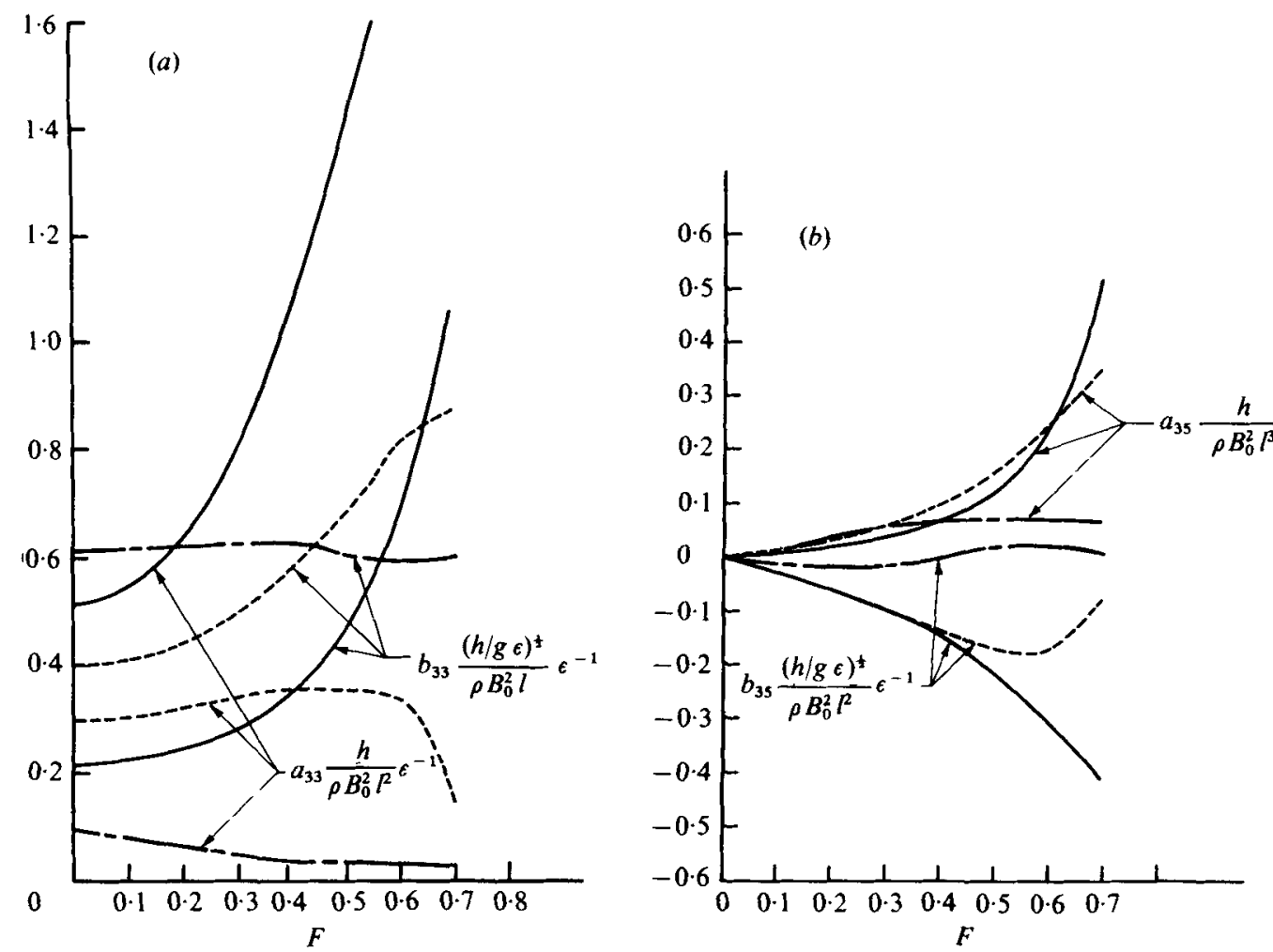

Figure 3. (a) Heave and $(b)$ heave-pitch added-mass and damping coefficients for ship with parabolic waterline of beam $\epsilon B_{0}$ and half-length $l$, in water of depth $\epsilon h$, plotted against Froude number $F .-, \omega l / c=0.5 ; \cdots, \omega l / c=1.0 ;-\cdots, \omega l / c=2.0$.

equations is therefore the same as the high frequency limit of the Tuck (1970) results. In this limit, the added-mass coefficients approach zero and the damping coefficients become constant. These results are physically unacceptable and indicate that the theory needs to be supplemented by a true high frequency theory such as that of $\operatorname{Kim}(1968)$.

\section{Sample problem}

Tuck (1970) presents heave and pitch added-mass and damping coefficients for zero forward speed for a symmetric hull of revolution with parabolic waterline

$$
B(x)=B_{0}\left(1-x^{2} / l^{2}\right) .
$$

For the general case of a forward speed and $\omega l / c$ [ $=\pi$ (ship length/wavelength)] of order 1 , the coefficients in $(33 a-h)$ for this geometry are displayed in figure 2 for values of the depth Froude number $F$ of $0,0 \cdot 3$ and $0 \cdot 6$. The high frequency limits discussed in the previous section are also indicated. The heave and cross-coupled coefficients are plotted against Froude number in figure 3 for values of the reduced frequency $\omega l / c$ of $0 \cdot 5,1 \cdot 0$ and $2 \cdot 0$. 
The effect of forward speed is clearly most significant in the low frequency range. It is interesting to note that the high frequency limits of the theory are approached at frequencies which normally can be considered to be $O(1)$.

This research was supported by the National Science Foundation under Grant no. ENG 74-20573.

\section{REFERENCES}

BeCK, R. F., SNYder, E. D. \& TUCK, E. O. 1974 Workshop on slender-body theory. Part II. Application to ship traffic control. Univ. Michigan, Dept. Naval Arch. Mar. Engng Rep. no. 164.

BECK, R. F. \& TuCK, E. O. 1971 Heave and pitch of ships in shallow water. 4th Austr. Conf. Hydrodyn. Fluid Mech., Monash Univ., Melbourne.

BECK, R. F. \& TUCK, E. O. 1972 Computation of shallow water ship motions. Proc. 9th ONR Symp. Naval Hydrodyn., Paris, France, p. 1543.

KIM, C. H. 1968 The influence of water depth on the heaving and pitching motions of a ship moving in longitudinal regular head waves. Schiffstech. 15, 127.

Lea, G. K. \& Feldman, J. P. 1972 Transcritical flow past slender ships. Proc. 9th ONR Symp. Naval Hydrodyn., Paris, France, p. 1527.

NAYFeh, A. H. 1973 Perturbation Methods. Wiley.

OGILvie, T. F. 1967 Nonlinear high-Froude number free-surface problems. J. Engng Math. 1, 215.

Plotkin, A. $1976 a$ The flow due to a slender ship moving over a wavy wall in shallow water. J. Engng Math. 10, 207.

PLotkin, A. $1976 b$ Hydrodynamic pressure field on a slender ship moving over a high-frequency wavy shallow bottom. J. Appl. Mech. 43, 232.

Trmman, R. \& Newman, J. N. 1962 The coupled damping coefficients of a symmetric ship. J. Ship Res. 5, 1.

TuCK, E. O. 1966 Shallow-water flows past slender bodies. J. Fluid Mech. 26, 81.

Tuck, E. O. 1970 Ship motions in shallow water. J. Ship Res. 14, 317.

Van Dyke, M. 1975 Perturbation Methods in Fluid Mechanics, 2nd edn. Parabolic Press. 